Discrete Comput Geom 37:409-417 (2007)

DOI: $10.1007 / \mathrm{s} 00454-006-1281-8$

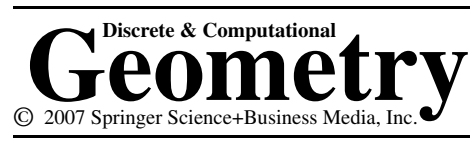

\title{
Line Transversals to Translates of Unit Discs
}

\author{
Jesús Jerónimo Castro \\ Centro de Investigación en Matemáticas, \\ Guanajuato, Gto., C.P. 36000, México \\ jeronimo@cimat.mx
}

\begin{abstract}
Let $\mathcal{F}$ be a family of convex figures in the plane. We say that $\mathcal{F}$ has property $T$ if there exists a line intersecting every member of $\mathcal{F}$. Also, the family $\mathcal{F}$ has property $T(k)$ if every $k$-membered subfamily of $\mathcal{F}$ has property $T$. Let $B$ be the unit disc centered at the origin. In this paper we prove that if a finite family $\mathcal{F}=\left\{x_{i}+B: i \in I\right\}$ of translates of $B$ has property $T(4)$ then the family $\mathcal{F}^{\prime}=\left\{x_{i}+\lambda B: i \in I\right\}$, where $\lambda=(1+\sqrt{5}) / 2$, has property $T$. We also give some results concerning families of translates of the unit disc which has either property $T(3)$ or property $T(5)$.
\end{abstract}

\section{Introduction}

Let $\mathcal{F}$ be a family of compact convex sets in the plane. We say that $\mathcal{F}$ has property $T$ if there exists a line intersecting every member of $\mathcal{F}$. Also, if $\mathcal{F}$ consist of at least $k$ members, we say that $\mathcal{F}$ has property $T(k)$ if every $k$-membered subfamily of $\mathcal{F}$ has property $T$.

Over the years considerable effort has been devoted to finding conditions on the family $\mathcal{F}$ such that $T(k)$ implies $T$. This problem was posed by Vincensini [9] and the first result in that direction was the following result due to Santaló [6]:

Santaló's Theorem. Let $\mathcal{F}$ be a family of parallelotopes in $\mathbb{E}^{n}$ with edges parallel to the coordinate axes. If $\mathcal{F}$ has property $T\left(2^{n-1}(2 n-1)\right)$ then $\mathcal{F}$ has property $T$.

In 1964 Grünbaum [3] posed the following problem:

Let $C$ be a centrally symmetric figure and let $\mathcal{F}=\left\{x_{i}+C\right\}$ be a finite family of translates of $C$ such that $\mathcal{F}$ has property $T(m)$. What is the smallest positive number $\lambda=\lambda(C, m)$ such that, for every such $\mathcal{F}$, the family $\mathcal{F}^{\prime}=\left\{x_{i}+\lambda C\right\}$ has property $T$ ? 
An upper bound for Grünbaum's problem was given by Eckhoff [2]:

Eckhoff's Theorem. If a finite family $\mathcal{F}$ of translates of $C$ in the plane has property $T(3)$ then there exists a parallel strip of $C$-width 1 intersecting all members of $\mathcal{F}$.

Where by the $C$-width of a set $X$, in direction $u$, we mean the ratio between the width of $X$ and the width of $C$, both taken in the direction $u$.

Heppes [5] proved the following result for the case of circles:

Heppes's Theorem. If a finite family $\mathcal{F}$ of pairwise disjoint translates of a disc of diameter 1 in the plane has property $T(3)$, then there exists a parallel strip of width $<0.65$ intersecting all members of $\mathcal{F}$.

The aim of this paper is to give some results in this direction for families of translates of unit circles.

\section{Some Results on Transversals}

We begin with some observations:

That three translates of the euclidean unit circle $(B), x_{i}+B(i=1,2,3)$, have a common line transversal is equivalent to saying that at least one altitude of the triangle $\triangle x_{1} x_{2} x_{3}$ has length at most 2 (see Fig. 1).

We can state the problem, for the case of unit circles, in the following equivalent way: Let $X$ be a finite set of points in the plane such that every three of them are contained in some parallel strip of width 2 . What is the smallest positive number $\alpha$ such that any such set $X$, with the above property, is contained in a parallel strip of width $\alpha$ ?

Independently, Eckhoff [1] and Dolnikov (1972) conjectured that this minimum number $\alpha$ must be equal to $1+\sqrt{5}$. This is equivalent to saying that

$$
\lambda(B, 3)=\frac{1+\sqrt{5}}{2} .
$$

We will show that if we consider property $T(4)$ instead of $T(3)$ the number $(1+\sqrt{5}) / 2$ is the best. That this number cannot be improved is shown by a classical example (which we call the pentagonal example) consisting of unit circles centered at the set of vertices of a regular pentagon whose sides have length equal to $2\left(\sin 72^{\circ}\right)^{-1}$ (Fig. 2).
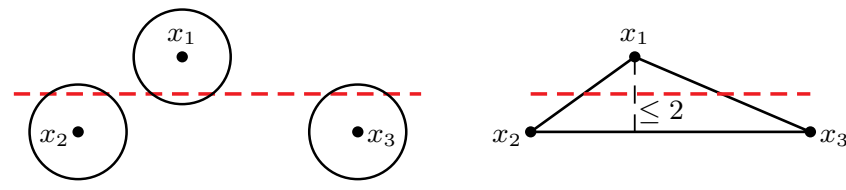

Fig. 1 


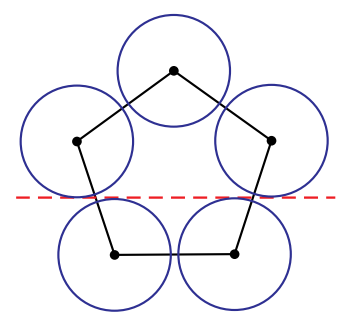

Fig. 2

In what follows, we consider only finite families of translates of a given convex figure. In order to prove Theorem 1 we will need the following lemma:

Lemma 1. Let $\mathcal{F}$ be a finite family of unit discs in the plane which has property $T(3)$, and let $\triangle x_{1} x_{2} x_{3}$ be a triangle of centers of the discs which has maximum area. If $\triangle x_{1} x_{2} x_{3}$ has two altitudes of length at most 2 , then there exists a parallel strip of width $1+\sqrt{5}$ which contains the set of centers.

Let $a, b, c$ be points in $\mathbb{E}^{2}$. We denote by $|a b|$ the length of the segment $[a, b]$ and by $d(a, b c)$ the distance from the point $a$ to the line $b c$. Also, if $C$ is a closed convex curve in the plane, we denote by $|C|$ the area of the convex hull of $C$. The following lemma is due to Straus [7]:

Straus's Lemma. If $C$ is a closed convex curve in $\mathbb{E}^{2}$ and one of the sides of an inscribed triangle $T$ of maximum area lies on $C$, then the ratio of the areas satisfies $\sqrt{5} \cdot|T| \geq|C|$, with equality if and only if $C$ is an affine regular pentagon.

In the case where $C$ is the boundary of a convex pentagon, it is not difficult to prove that there is a triangle, $T$, of maximum area whose vertices are vertices of the pentagon. Thus, one of the sides of $T$ lies on $C$ and so Straus's lemma applies.

Proof of Lemma 1. Obviously, there exists such a triangle $\triangle x_{1} x_{2} x_{3}$ since the set of centers, $X$, is finite. Suppose that $d\left(x_{2}, x_{1} x_{3}\right) \leq 2$ and $d\left(x_{3}, x_{1} x_{2}\right) \leq 2$. Let $\triangle y_{1} y_{2} y_{3}$ be the homothetic copy of $\triangle x_{1} x_{2} x_{3}$ with center of homothety at the centroid of $\triangle x_{1} x_{2} x_{3}$ and a constant of homothety equal to -2 . We have $X \subset \Delta y_{1} y_{2} y_{3}$, otherwise we can find another triangle with vertices at points of $X$ with greater area. Now, let $p: \mathbb{E}^{2} \longrightarrow \mathbb{E}^{2}$ be an affine transformation with $a_{i}=p\left(x_{i}\right)$, for $i=1,2,3$, such that $\left|a_{1} a_{2}\right|=\left|a_{1} a_{3}\right|$, $\left|a_{1} a_{2}\right| /\left|a_{2} a_{3}\right|=(1+\sqrt{5}) / 2$, and $\left|a_{2} a_{3}\right|=2\left(\sin 72^{\circ}\right)^{-1}$. Also, let $b_{i}=p\left(y_{i}\right)$, for $i=1,2,3$, and $X^{\prime}=p(X)$. Clearly, $\triangle a_{1} a_{2} a_{3}$ is a triangle of $X^{\prime}$ of maximum area.

Let $m n$ be a line parallel to $a_{1} a_{2}$ and let $o q$ be parallel to $a_{1} a_{3}$, such that $d\left(n, b_{1} b_{2}\right)=$ $d\left(q, b_{1} b_{3}\right)=1+\sqrt{5}$ (see Fig. 3). Then $X^{\prime}$ must be contained either in the quadrilateral $m n b_{1} b_{2}$ or in the quadrilateral $q o b_{3} b_{1}$. Otherwise, there exist points $d, e \in X^{\prime}$ such that 


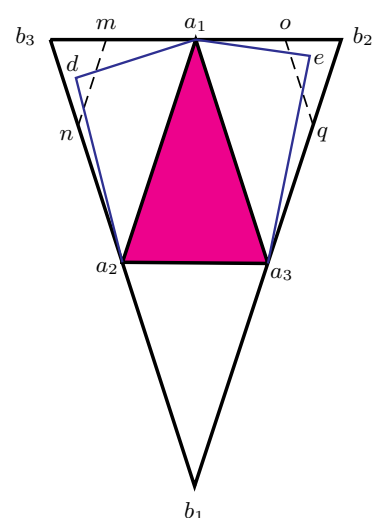

Fig. 3

$d\left(e, b_{1} b_{3}\right)>1+\sqrt{5}$ and $d\left(d, b_{1} b_{2}\right)>1+\sqrt{5}$. In this case we have

$$
\frac{\left|a_{1} a_{2} a_{3}\right|}{\left|a_{1} d a_{2} a_{3} e\right|}<\frac{1}{\sqrt{5}},
$$

contradicting Straus's lemma. Then, without loss of generality, we can assume that $X^{\prime}$ is contained in the quadrilateral $m n b_{1} b_{2}$. Since the strip with boundary lines $m n$ and $b_{1} b_{2}$ contains $X^{\prime}$, it follows that the strip with boundary lines $p^{-1}(m n)$ and $p^{-1}\left(b_{1} b_{2}\right)$ contains $X$ and this strip has width at most $1+\sqrt{5}$.

Now, as we have said before, by replacing $T(3)$ by $T(4)$ we obtain the following result which was conjectured by Eckhoff [1]:

Theorem 1. Let $\mathcal{F}$ be a finite family of unit discs in the plane. If $\mathcal{F}$ has property $T(4)$ then there exists a parallel strip of width $1+\sqrt{5}$ which contains the set of centers.

Proof of Theorem 1. Let $\triangle x_{1} x_{2} x_{3}$, where $\left\{x_{1}, x_{2}, x_{3}\right\} \subset X$, be a triangle of maximum area. By Lemma 1 we may assume that $\triangle x_{1} x_{2} x_{3}$ has exactly one altitude $\leq 2$, so we may assume $(1+\sqrt{5}) / 2<d\left(x_{2}, x_{1} x_{3}\right) \leq 2$.

As in the proof of Lemma 1 we have that $X \subset \Delta y_{1} y_{2} y_{3}$, where $\triangle y_{1} y_{2} y_{3}$ is the homothetic copy of $\triangle x_{1} x_{2} x_{3}$ with center of homothety the centroid of $\triangle x_{1} x_{2} x_{3}$ and constant of homothety equal to -2 . Clearly, there must exist an $x \in X$ with $d\left(x, y_{1} y_{3}\right)>$ $1+\sqrt{5}$, or else we are done, so let $e \in X$ be a point that maximizes this distance (see Fig. 4).

Since every four points of $X$ are contained in a strip of width 2 and the triangle $\triangle x_{1} x_{2} x_{3}$ has exactly one altitude $\leq 2$, we have either $d\left(x_{2}, x_{3} e\right) \leq 2$ or $d\left(x_{2}, x_{1} e\right) \leq 2$. Without loss of generality we may assume that $d\left(x_{2}, x_{3} e\right) \leq 2$.

Claim 1. There exists a parallel strip which contains $X$, whose boundary lines are parallel to $x_{3}$ e and whose width is less than $1+\sqrt{5}$. 


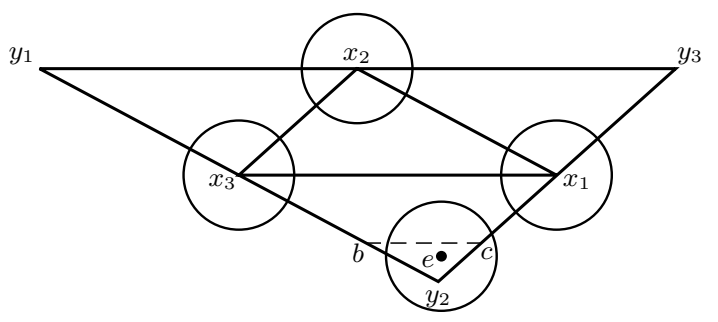

Fig. 4

Proof of Claim 1. Let $p: \mathbb{E}^{2} \longrightarrow \mathbb{E}^{2}$ be an affine transformation with $x_{i}^{\prime}=p\left(x_{i}\right)$, for $i=1,2,3$, such that $\left|x_{1}^{\prime} x_{2}^{\prime}\right|=\left|x_{1}^{\prime} x_{3}^{\prime}\right|,\left|x_{1}^{\prime} x_{2}^{\prime}\right| /\left|x_{2}^{\prime} x_{3}^{\prime}\right|=(1+\sqrt{5}) / 2$, and $\left|x_{2}^{\prime} x_{3}^{\prime}\right|=$ $2\left(\sin 72^{\circ}\right)^{-1}$. Also, let $y_{i}^{\prime}=p\left(y_{i}\right)$, for $i=1,2,3, e^{\prime}=p(e)$, and $X^{\prime}=p(X)$. We consider points $f \in\left[x_{3}^{\prime}, y_{2}^{\prime}\right], g \in\left[x_{1}^{\prime}, y_{2}^{\prime}\right], p \in\left[x_{2}^{\prime}, y_{3}^{\prime}\right]$, and $q \in\left[x_{1}^{\prime}, y_{3}^{\prime}\right]$, such that $d\left(f, y_{1}^{\prime} y_{3}^{\prime}\right)=d\left(g, y_{1}^{\prime} y_{3}^{\prime}\right)=d\left(p, y_{1}^{\prime} y_{2}^{\prime}\right)=d\left(q, y_{1}^{\prime} y_{2}^{\prime}\right)=1+\sqrt{5}$ (see Fig. 5). Let $m \in\left[f, y_{2}^{\prime}\right]$ and $n \in\left[g, y_{2}^{\prime}\right]$ be points such that $m n$ is parallel to $y_{1}^{\prime} y_{3}^{\prime}$ and $e^{\prime} \in[m, n]$. We deduce $X^{\prime}$ must be contained in the quadrilateral $m n y_{3}^{\prime} y_{1}^{\prime}$. Now, we consider points $r \in\left[x_{2}^{\prime}, p\right], s \in\left[x_{1}^{\prime}, q\right]$, such that $r s$ is parallel to $y_{1}^{\prime} y_{2}^{\prime}$ and $|q s|=|g n|$. Since $\triangle x_{1}^{\prime} x_{2}^{\prime} x_{3}^{\prime}$ is a triangle of maximum area we know by Straus's lemma that any point of $X^{\prime}$ must be below the line $r s$. This implies that $X^{\prime}$ must be contained in the pentagon $y_{1}^{\prime} m n s r$. Let $S_{1}$ be the parallel strip whose boundary lines are $l_{1}$ and $l_{2}$, with $m \in l_{1}, r \in l_{2}$, and $l_{1}$ and $l_{2}$ parallel to $x_{3}^{\prime} e^{\prime}$. We then have $X^{\prime} \subset S_{1}$ which implies that $X \subset p^{-1}\left(S_{1}\right)$. Now, we will prove that $p^{-1}\left(S_{1}\right)$ has width less than $1+\sqrt{5}$.

Let $t$ be the point of segment $[p, q]$ such that $|p t|=|p r|=|f m|$ (see Fig. 6). Thus the segments $[t, m],[p, f]$, and $\left[x_{2}^{\prime}, x_{3}^{\prime}\right]$ are parallel. Since $\left|x_{1}^{\prime} g\right|>\frac{1}{2}\left|x_{1}^{\prime} y_{2}^{\prime}\right|$ it follows that $\angle x_{1}^{\prime} x_{3}^{\prime} e^{\prime}>\frac{1}{2} \angle x_{1}^{\prime} x_{3}^{\prime} y_{2}^{\prime}=\angle p r t$. This in turn implies that $l_{2}$ intersects the interior of

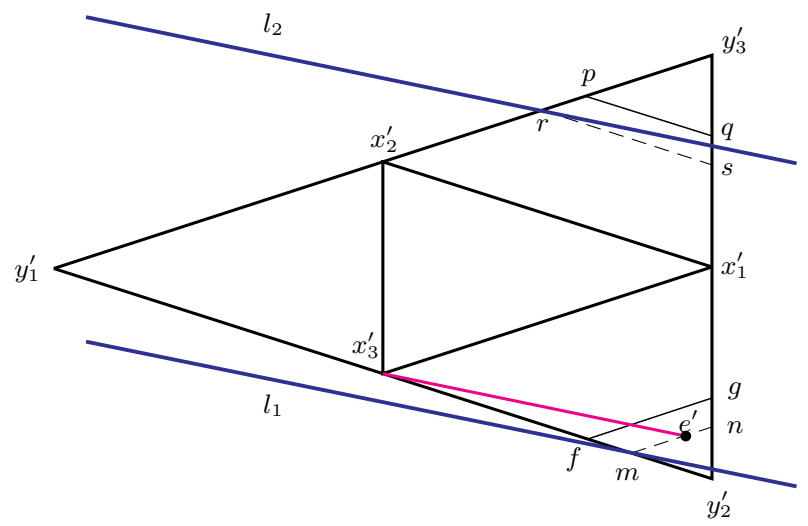

Fig. 5 


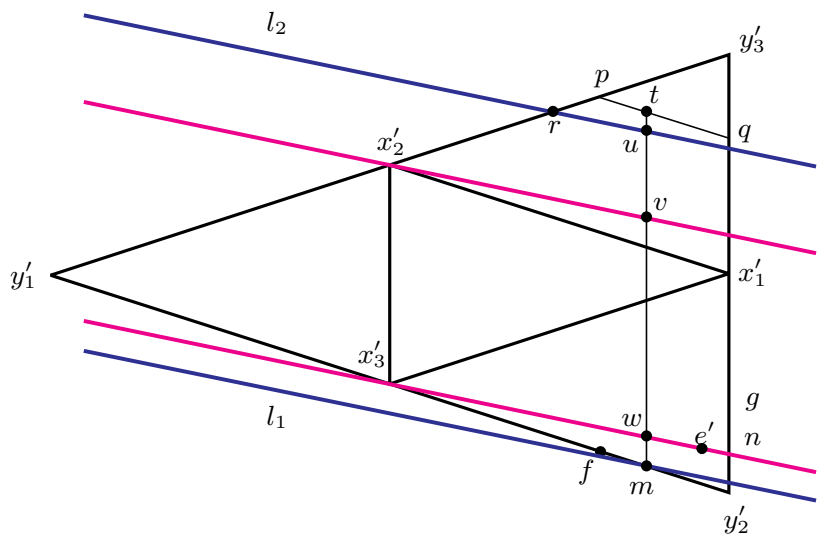

Fig. 6

the segment $[t, m]$ in a point $u$. Moreover, let $x_{2}^{\prime} v$ be parallel to $x_{3}^{\prime} e^{\prime}$, with $v \in[t, m]$, and let $w=\left[x_{3}^{\prime}, e^{\prime}\right] \cap[t, m]$.

Let $S_{2}$ be the strip whose boundary lines are $x_{2}^{\prime} v$ and $x_{3}^{\prime} w$. Since $|v w|=\left|x_{2}^{\prime} x_{3}^{\prime}\right|=$ $\left|x_{1}^{\prime} y_{2}^{\prime}\right|,|t m|=\left|q y_{2}^{\prime}\right|$, and $\left|q y_{2}^{\prime}\right| /\left|x_{1}^{\prime} y_{2}^{\prime}\right|=(1+\sqrt{5}) / 2$, we have

$$
\frac{|u m|}{|v w|}<\frac{|t m|}{|v w|}=\frac{\left|q y_{2}^{\prime}\right|}{\left|x_{1}^{\prime} y_{2}^{\prime}\right|}=\frac{1+\sqrt{5}}{2}
$$

this implies that the ratio between the width of $S_{1}$ and the width of $S_{2}$ is less than $(1+\sqrt{5}) / 2$. As affine transformations preserve the ratio between lengths of parallel segments, then we get that the ratio between the width of the strip $p^{-1}\left(S_{1}\right)$ and the width of the strip $p^{-1}\left(S_{2}\right)$ is less than $(1+\sqrt{5}) / 2$. Since the width of $p^{-1}\left(S_{2}\right)$ is at most 2 (since $d\left(x_{2}, x_{3} e\right) \leq 2$ ), the width of $p^{-1}\left(S_{1}\right)$ is less than $1+\sqrt{5}$.

Thus, we conclude that there exists a parallel strip of width $1+\sqrt{5}$ which contains $X$.

Remark 1. Notice that in the proof of Theorem 1 we only require that the translates of $B$ by the vectors $x_{1}, x_{2}, x_{3}$, and $e$ possess a common transversal, i.e., it is not necessary that the whole family $\mathcal{F}$ possesses the property $T(4)$. Furthermore, since we know that the number $(1+\sqrt{5}) / 2$ is necessary for the pentagonal example, we have proved that $\lambda(B, 4)=(1+\sqrt{5}) / 2$.

With stronger conditions it is possible to obtain a better constant, $2 \sqrt{2}$, although this constant could probably be reduced. We obtain this result by a nice application of the following theorem due to Hadwiger and Debrunner [4]:

Hadwiger-Debrunner's Theorem. Given any family of parallelograms with parallel edges, such that any three can be intersected by an ascending line, there exists an ascending line intersecting all the parallelograms. 


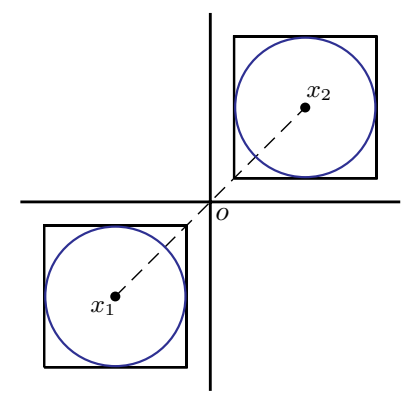

Fig. 7

Theorem 2. Let $\mathcal{F}$ be a family of unit discs in the plane which has property $T(5)$. Then there exists a parallel strip of width $2 \sqrt{2}$ which contains the set of centers.

Proof of Theorem 2. Let $x_{1}, x_{2}$ be points in the set of centers, $X$, which are further apart and let $o$ be the midpoint of the segment $\left[x_{1}, x_{2}\right]$. If $d\left(x_{1}, x_{2}\right) \leq 2 \sqrt{2}$, then for every point $x \in X$ we have that the distance from $x$ to the line perpendicular to $x_{1} x_{2}$ through $o$ is $\leq \sqrt{2}$. So the conclusion of the theorem follows. Else, we may assume that $d\left(x_{1}, x_{2}\right)>2 \sqrt{2}$. Consider the coordinate axes through $o$ in such a way that $x_{1} x_{2}$ is an angle-bisector to the angle formed by the axes (see Fig. 7). With each disc in $\mathcal{F}$ we associate a circumscribed square which has sides parallel to the coordinate axes; in this way we obtain a finite family, $F^{\prime}$, of translates of a square with side 2 .

As we can see, the squares centered at $x_{1}$ and $x_{2}$ have only ascending transversals. Furthermore, since $\mathcal{F}$ has property $T(5)$, and so $\mathcal{F}^{\prime}$, we have that every three members of $\mathcal{F}^{\prime}$ have an ascending transversal. It follows, by Hadwiger-Debrunner's theorem that there exists a line $l$ which is a common transversal to every member of $\mathcal{F}^{\prime}$. Therefore, any center of a square belonging to $\mathcal{F}^{\prime}$ has a distance at most $\sqrt{2}$ from $l$. We conclude that there exists a parallel strip of width $2 \sqrt{2}$ which contains the set of centers.

Now, denote by $Q$ a square of side 2 and consider a family $\mathcal{F}$ of translates of $Q$. If $\mathcal{F}$ has property $T(6)$ then there exists a line transversal to each member of this family, as was shown by Santaló. Assume that the family has property $T(3)$. Then we obtain that the constant 1 in Eckhoff's result is the best possible. The following result was previously proved by Eckhoff [2], however, for completeness we give a proof for it here:

Theorem 3. Let $\mathcal{F}$ be a finite family of translates of $Q$ which has property $T$ (3). Then there exists a parallel strip of $Q$-width 1 which intersects every member of $\mathcal{F}$. Moreover, this constant cannot be reduced, i.e., $\lambda(Q, 3)=2$.

Proof of Theorem 3. By Eckhoff's theorem we know that there exists a parallel strip of $Q$-width at most 2 which contains the set of centers. Let $\mathcal{F}=\left\{P_{1}, P_{2}, P_{3}, P_{4}\right\}$ be a family consisting of four translates of the unit square, where the translates have centers $x_{1}, x_{2}, x_{3}$, and $x_{4}$, respectively. Choose the translates so that $x_{1} x_{2} x_{3} x_{4}$ is a square of side 


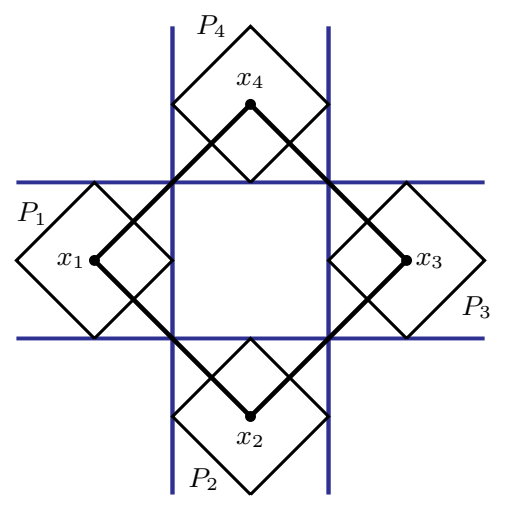

Fig. 8

2 whose sides are parallel to the sides of the unit square. As we can see in Fig. 8, $\mathcal{F}$ has property $T$ (3) but no property $T$, i.e., there exists no common transversal for the family $\mathcal{F}$. Since any parallel strip containing the set $\left\{x_{1}, x_{2}, x_{3}, x_{4}\right\}$ must contain the square $x_{1} x_{2} x_{3} x_{4}$, it must have $Q$-width at least 2, therefore, the number 1 given in the theorem cannot be reduced.

Remark 2. Let $K$ be a convex body in the plane. The following was observed by Eckhoff:

$$
\lambda(K, 3)=2 \quad \text { if and only if } K \text { is a parallelogram. }
$$

\section{Final Remarks}

The Eckhoff-Dolnikov conjecture remains open for the case where the triangle of maximum area has only one altitude $\leq 2$. It is possible that the techniques used in this paper could be useful for the proof of this conjecture.

Letting $\mathcal{F}$ be a finite family of translates of a figure of constant width, we find that Lemma 1, Theorem 1, and Theorem 2 are still valid. This can be seen from the following reduction noted by Tverberg [8]: Let $K$ be a convex set and let $K^{\prime}$ be the centrally symmetric set obtained by setting $K^{\prime}=\frac{1}{2}(K+(-K))$. Let $\mathcal{F}=\left\{x_{i}+K: i \in I\right\}$ be a family of translates of $K$ and let $\mathcal{F}^{\prime}=\left\{x_{i}+K^{\prime}: i \in I\right\}$ be the associated family of translates of $K^{\prime}$. Then it is easily seen that $\mathcal{F}$ and $\mathcal{F}^{\prime}$ share the same properties with respect to disjointness, transversals, and GPs. We know that in the case when $K$ is a figure of constant width, $K^{\prime}$ is a euclidean disc, hence our assertion follows.

Obviously, the number $2 \sqrt{2}$ in Theorem 2 could be reduced using better arguments. 


\section{Acknowledgements}

I thank V.L. Dolnikov for his constant advice and many helpful discussions. I am also indebted to Jürgen Eckhoff and the unknown referee for enhancing the readability of the paper.

\section{References}

1. J. Eckhoff (1969), Transversalenprobleme vom Gallai'schen Typ, Dissertation, Göttingen.

2. J. Eckhoff (1973), Transversalenprobleme in der Ebene, Arch. Math. 24, 191-202.

3. B. Grünbaum (1964), Common secants for families of polyhedra, Arch. Math. 15, 76-80.

4. H. Hadwiger and H. Debrunner (1955), Ausgewählte einzelprobleme der kombinatorischen geometrie in der ebene, Enseign. Math. 1, 56-89.

5. A. Heppes (2005), New upper bound on the transversal width of T(3)-families of discs, Discrete Comput. Geom. 34, 463-474.

6. L. Santaló (1940), Un teorema sobre conjuntos de paralelepípedos de aristas paralelas, Publ. Inst. Mat. Univ. Nac. Litoral 2, 49-60.

7. E.G. Straus (1978), Some extremal problems in combinatorial geometry, Lecture Notes in Mathematics, vol. 686, pp. 308-312.

8. H. Tverberg (1989), Proof of Grünbaum's conjecture on common transversals for translates, Discrete Comput. Geom. 4, 191-203.

9. P. Vincensini (1935), Figures convexes et variétés linéaires de l'espace euclidien à $n$ dimensions, Bull. Sci. Math. 59, 163-174.

Received February 2005, and in revised form February 28, 2006. Online publication March 9, 2007. 